\title{
Phase coexistence and hysteresis effects in the pressure-temperature phase diagram of $\mathrm{NH}_{3} \mathrm{BH}_{3}$
}

\author{
Ove Andersson, ${ }^{1}$ Yaroslav Filinchuk, ${ }^{2,3}$ Vladimir Dmitriev, ${ }^{2}$ Issam Quwar, ${ }^{1}$ Alexandr V. Talyzin, ${ }^{1}$ and Bertil Sundqvist ${ }^{1, *}$ \\ ${ }^{1}$ Department of Physics, Umeå University, SE-90187 Umeå, Sweden \\ ${ }^{2}$ Swiss-Norwegian Beam Lines at ESRF, B.P. 220, F-38043 Grenoble, France \\ ${ }^{3}$ Institute of Condensed Matter and Nanosciences, Université Catholique de Louvain, B-1348 Louvain-la-Neuve, Belgium
}

(Received 3 March 2011; revised manuscript received 3 May 2011; published 15 July 2011)

\begin{abstract}
The potential hydrogen storage compound $\mathrm{NH}_{3} \mathrm{BH}_{3}$ has three known structural phases in the temperature and pressure ranges $110-300 \mathrm{~K}$ and $0-1.5 \mathrm{GPa}$, respectively. We report here the boundaries between, and the ranges of stability of, these phases. The phase boundaries were located by in situ measurements of the thermal conductivity, while the actual structures in selected areas were identified by in situ Raman spectroscopy and x-ray diffraction. Below 0.6 GPa, reversible transitions involving only small hysteresis effects occur between the room-temperature tetragonal plastic crystal $I 4 \mathrm{~mm}$ phase and the low-temperature orthorhombic Pmn $2_{1}$ phase. Transformations of the $I 4 \mathrm{~mm}$ phase into the high-pressure orthorhombic $C m c 2_{1}$ phase, occurring above $0.8 \mathrm{GPa}$, are associated with very large hysteresis effects, such that the reverse transition may occur at up to $0.5 \mathrm{GPa}$ lower pressures. Below $230 \mathrm{~K}$, a fraction of the $C m c 2_{1}$ phase is metastable to atmospheric pressure, suggesting the possibility that dense structural phases of $\mathrm{NH}_{3} \mathrm{BH}_{3}$, stable at room temperature, could possibly be created and stabilized by alloying or by other methods. Mixed orthorhombic $P m n 2_{1} / C m c 2_{1}$ phases were observed in an intermediate pressure-temperature range, but a fourth structural phase predicted by Filinchuk et al. [Phys. Rev. B 79, 214111 (2009)] was not observed in the pressure-temperature ranges of this experiment. The thermal conductivity of the plastic crystal $14 \mathrm{~mm}$ phase is about $0.6 \mathrm{~W} \mathrm{~m} \mathrm{~W}^{-1} \mathrm{~K}^{-1}$ and only weakly dependent on temperature, while the ordered orthorhombic phases have higher thermal conductivities limited by phonon-phonon scattering.
\end{abstract}

DOI: 10.1103/PhysRevB.84.024115

PACS number(s): 81.30.Dz, 62.50.-p, 65.40.-b, 88.30.R-

\section{INTRODUCTION}

Over the last decade there has been a vast and growing interest in the possibility of storing hydrogen in the form of solid, hydrogen-rich compounds. Such materials may contain a larger amount of hydrogen per unit volume than solid or liquid hydrogen itself, making compact hydrogen storage for mobile applications possible. ${ }^{1}$ Apart from the important issues of reversibility and environmental impact, a crucial problem is to optimize the volumetric concentration of hydrogen for a given compound. It is thus desirable to find structures with very high densities, and as a result there has recently been a very large interest in the structural properties of candidate materials such as complex hydrides. In particular, the highpressure structural properties of many such materials have been explored by both experiments ${ }^{2-5}$ and calculations $s^{3-6}$ in the quest for highly dense phases which, ideally, might be stabilized in metastable form under atmospheric, or at least technologically safe, pressures.

Ammonia-borane, $\mathrm{NH}_{3} \mathrm{BH}_{3}$, contains $19.6 \%$ hydrogen by weight and is thus an excellent candidate as an efficient hydrogen storage material. ${ }^{7}$ The hydrogen can be released by thermolysis, starting at temperatures ${ }^{7-10}$ already above about $80^{\circ} \mathrm{C}$, but at higher temperatures the process also tends to release other volatile gases such as borazine, and regeneration is difficult. ${ }^{7,9}$ Closely related compounds or complexes containing alkali or other metals have been found to have significantly improved hydrogen release properties ${ }^{7}, 10-12$ and may even allow rehydrogenation. ${ }^{11}$

Over the last decade, the high-pressure behavior and properties of $\mathrm{NH}_{3} \mathrm{BH}_{3}$ have been studied by several groups. ${ }^{13-24}$ The earliest studies ${ }^{13,14}$ were basically motivated by the anomalous lattice stability of $\mathrm{NH}_{3} \mathrm{BH}_{3}$, which is a solid at room temperature in contrast to the very similar compounds diborane, $\mathrm{B}_{2} \mathrm{H}_{6}$ (liquid ${ }^{25}$ up to $4 \mathrm{GPa}$ ) and ethane, $\mathrm{C}_{2} \mathrm{H}_{6}$ (gas). The reason for this stability is believed to be the existence of dihydrogen bonds between $\mathrm{NH}_{3} \mathrm{BH}_{3}$ molecules. ${ }^{26}$ Raman studies of these bonds under pressure ${ }^{13,14}$ also revealed the existence of structural transformations at pressures between 0.5 and $1.4 \mathrm{GPa}$. Later in situ studies, using both $\mathrm{IR}^{18}$ and Raman ${ }^{15,16,18,19}$ spectroscopy as well as x-ray ${ }^{17,20,21}$ and neutron ${ }^{20}$ diffraction, have provided further information about the structural evolution of $\mathrm{NH}_{3} \mathrm{BH}_{3}$ with pressure at room temperature. Near atmospheric pressure at room temperature $\mathrm{NH}_{3} \mathrm{BH}_{3}$ has a plastic crystal ${ }^{10}$ phase with aligned but rotationally disordered molecules forming a tetragonal structure ${ }^{7,17,27}$ with symmetry $14 \mathrm{~mm}$. This phase transforms near $1.1 \mathrm{GPa}$ into an orthorhombic structure $e^{17,20,21}$ with symmetry $C m c 2_{1}$. The transition is associated with the anomalies reported in Raman spectra in the range $0.5-2 \mathrm{GPa}$ in several papers. ${ }^{13-16,18,19} \mathrm{~A}$ further increase in pressure above $2 \mathrm{GPa}$ has been reported to induce further structural changes, but in this pressure range there is no consensus on actual transition pressures and structures. Filinchuk et al. ${ }^{17}$ report no further phase changes up to $12 \mathrm{GPa}$, while other authors report anomalies at pressures near $5,{ }^{15,16,18,19,21} 8,{ }^{18,20}$ and $10-12^{15,16,18} \mathrm{GPa}$. Chen et $a .^{21}$ find an isostructural second-order transition, with a significant increase in bulk modulus, near $5 \mathrm{GPa}$, while Kumar et al. ${ }^{20}$ identify the phase formed at $8 \mathrm{GPa}$ as a triclinic structure with symmetry $P_{1}$. A recent theoretical calculation ${ }^{24}$ instead suggests a transformation back to $14 \mathrm{~mm}$ near $12 \mathrm{GPa}$. High-temperature, high-pressure studies ${ }^{19,22}$ of the thermal decomposition of $\mathrm{NH}_{3} \mathrm{BH}_{3}$ have also been carried out, and it has recently been observed that significant amounts of molecular $\mathrm{H}_{2}$ may be inserted into the $\mathrm{NH}_{3} \mathrm{BH}_{3}$ lattice at very high pressures. ${ }^{16,22,23}$ 
At atmospheric pressure, tetragonal $14 m m \mathrm{NH}_{3} \mathrm{BH}_{3}$ transforms on cooling below ${ }^{17,28-31} 218-225 \mathrm{~K}$ into another orthorhombic phase with symmetry Pmn $2_{1}$. While in the $I 4 m m$ phase the molecular axes are aligned with the lattice $c$ axis, the molecular axes become inclined relative to this in the two orthorhombic phases. In the low-temperature $P m n 2_{1}$ phase the angle is rather small and depends on temperature, ${ }^{17,31}$ but in the high-pressure $C m c 2_{1}$ phase it is large, $69^{\circ}$ near the $14 m m-C m c 2_{1}$ transition $^{17}$ and increasing to $79^{\circ}$ at $4.65 \mathrm{GPa}$. Also, while the $I 4 m m-P m n 2_{1}$ transformation is ${ }^{17}$ "quite close to second order" and associated with a very small volume change $(0.27 \%)$ and a narrow range of coexistence at atmospheric pressure, ${ }^{17,30}$ the high-pressure $14 \mathrm{~mm}-\mathrm{Cmc} 2_{1}$ transformation is reported ${ }^{17}$ to have a significant range of coexistence and a large hysteresis in the transition pressure. This transition is also associated with a significant volume change, reported as ${ }^{17} 4.4 \%$ to $^{21} 6 \%$. The transition and structures observed at atmospheric pressure have been studied by x-ray diffraction (XRD), ${ }^{17}$ NMR, ${ }^{29,32}$ vibrational spectroscopy, ${ }^{30}$ and elastic ${ }^{31}$ and inelastic ${ }^{33}$ neutron scattering and appear to be reasonably well understood. However, since the two known phase transformations seem to have quite different characters, in spite of the obvious structural similarities between the two orthorhombic phases, further high-pressure studies of the properties of $\mathrm{NH}_{3} \mathrm{BH}_{3}$ are clearly needed.

In this paper we report the results of a detailed study of the pressure-temperature $(p-T)$ phase diagram of $\mathrm{NH}_{3} \mathrm{BH}_{3}$ at temperatures $(T)$ in the range 110-300 $\mathrm{K}$ and in the pressure $(p)$ range between atmospheric pressure and 1.5 GPa. We have identified the positions of the phase boundaries between the three known phases discussed above using thermal conductivity measurements, and we have tried to find a fourth structural phase, predicted by Filinchuk et $a l .{ }^{17}$ to exist in the low temperature area of the phase diagram. While we find no evidence for the latter phase, we have successfully mapped the phase diagram down to $110 \mathrm{~K}$ in the range of pressure investigated and used Raman scattering and XRD to identify an unknown phase appearing in an intermediate pressure-temperature range as a mixture of the two orthorhombic structures. We also show that the high-pressure $C m c 2_{1}$ phase is strongly metastable and can be partially recovered at atmospheric pressure below $230 \mathrm{~K}$, hinting at possibilities to create dense, metastable, hydrogenrich structures based on $\mathrm{NH}_{3} \mathrm{BH}_{3}$.

\section{EXPERIMENTAL DETAILS}

The $\mathrm{NH}_{3} \mathrm{BH}_{3}$ material studied was obtained from SigmaAldrich, had a nominal purity greater than $97 \%$, and was used without further purification. The sample source and quality were intentionally identical to those used by Filinchuk et al. ${ }^{17}$ such that a direct comparison should be possible between the two sets of results. Although $\mathrm{NH}_{3} \mathrm{BH}_{3}$ should not be very sensitive to air or humidity, ${ }^{9,10}$ all samples were stored and handled in a glove-box under dry Ar gas.

The thermal conductivity, $\kappa$, of our samples was measured using a well-known dynamic hot-wire method, ${ }^{34}$ in which $\kappa$ is basically calculated from the rate of temperature rise in a thin $\mathrm{Ni}$ wire probe inserted into the bulk of the sample and heated by a short (about $1 \mathrm{~s}$ ) power pulse. The wire acts as both heater and sensor for the temperature rise, which is calculated from the measured relation between the wire resistance and temperature. Provided that the wire is in good thermal contact with the surrounding sample, and that the sample is physically large enough and the thermal diffusivity low enough that temperature waves reflected from the outer boundaries of the sample do not have time to interfere with the original temperature wave created by the heat pulse, the inaccuracy of this method is estimated to be smaller than $\pm 2 \%$. Indeed, in our experience, measurements on different samples of the same material under identical conditions usually differ by $\pm 1 \%$ or less, provided the structural state (average grain size, orientation, etc.) is the same. (However, it should be noted that phase transformations and treatment under different $p-T$ conditions may lead to somewhat different final states.) In the present experiments, the magnitude of $\kappa$ in the orthorhombic states has been observed to differ by at most $8 \%$ at the same pressure-temperature coordinates, depending on the transformation coordinates used and the $p-T$ path traveled by the sample. Phase transformations are usually easily detected by the step changes in $\kappa$ and $d \kappa / d T$ (or $d \kappa / d p$ ) associated with most structural changes. Because of the dynamic character of the method, exothermal or endothermal effects add further experimental artifacts to the measured data, helping to identify transitions. The method has already been used in studies of the high-pressure phase diagrams of several other complex hydrides. ${ }^{3,4,35-38}$ In addition to thermal conductivity data, the method also provides data for the specific heat per unit volume, although with lower accuracy.

The thermal measurements were carried out using a largevolume all-steel piston-and-cylinder device with an internal diameter of $45 \mathrm{~mm}$ and a maximum pressure capacity of $3 \mathrm{GPa}$. The samples were encased in Teflon cells with 5-mm-thick walls. The device was thermally insulated using mineral wool and could be heated electrically or cooled by spraying with liquid nitrogen. The pressure was calculated from the applied press load using an empirical calibration function, and the temperature of the sample was measured by a Chromel-Alumel thermocouple which had been calibrated against a commercially available silicon diode thermometer.

High-pressure Raman and XRD measurements were carried out using a diamond anvil cell with $0.5-0.7-\mathrm{mm}$ culets and beryllium seats. Samples were contained by stainless steel gaskets with holes $0.3 \mathrm{~mm}$ in diameter, and the pressure was calculated from the shift in the fluorescence from added ruby balls. A thermocouple was attached directly to the gasket to measure the temperature, and the system was cooled using a liquid nitrogen-gas flow system. XRD patterns were recorded at the Swiss-Norwegian Beam Lines (BM1A) at the European Synchrotron Radiation Facility (ESRF) in Grenoble using synchrotron radiation with a wavelength $\lambda=0.7092 \AA$ and a MAR345 image plate detector. The two-dimensional XRD patterns were integrated using Fit2D software. Raman spectra were obtained using a Renishaw 1000 Raman spectrometer with excitation wavelengths of 514 or $633 \mathrm{~nm}$, using a long-focus $50 \times$ objective.

Both types of pressure cells were filled in the glove-box mentioned above, and in neither case did we use a pressuretransmitting medium. Because of the low shear strength of $\mathrm{NH}_{3} \mathrm{BH}_{3}$ quasi-hydrostatic conditions should still prevail 
in the pressure and temperature ranges studied here, and no problems connected with pressure gradients have been identified in our earlier studies on complex hydrides ${ }^{3,4,35-38}$ or other soft materials ${ }^{39}$ using the same equipment and methods.

\section{EXPERIMENTAL RESULTS AND DISCUSSION}

The primary aims of this work were to map the pressuretemperature phase diagram of $\mathrm{NH}_{3} \mathrm{BH}_{3}$ up to about $1.5 \mathrm{GPa}$ at room temperature and below, a range where three well-defined structural phases are known to exist, and to search for a fourth structural phase predicted by Filinchuk et al. ${ }^{17}$ to appear at low temperatures. We therefore measured the thermal conductivity $\kappa$ of two samples of $\mathrm{NH}_{3} \mathrm{BH}_{3}$ as a function of $T$ and $p$ during a large number of (approximately) isothermal pressure runs and isobaric temperature cycles. Such measurements give a detailed map of the phase boundaries, but to identify the actual structure(s) in particular areas we also carried out complementary studies by Raman spectroscopy and XRD.

The measurements of $\kappa$ on the first sample showed a number of phase transformation anomalies which seemed to define a continuously curving phase boundary connecting the known low-temperature $I 4 m m-P m n 2_{1}$ transition with the known high-pressure $14 m m-C m c 2_{1}$ transition. At intermediate pressures near $0.7 \mathrm{GPa}$, however, temperature cycling revealed anomalous values for $\kappa$ on heating into the $14 \mathrm{~mm}$ phase. Unfortunately, before we could investigate this matter in detail, the hot-wire probe broke during a $P m n 2_{1}$ to $I 4 \mathrm{~mm}$ transformation under isothermal conditions. To identify the structures present in the anomalous region we then carried out a series of in situ Raman studies and XRD measurement. The results obtained clearly showed that, in this pressure range, cooling through the phase boundary produced a mixture of the two orthorhombic phases, and that no new structural phase was involved. Further measurements of $\kappa$ were then carried out on a second sample in order to understand the puzzling hysteresis behavior sometimes observed in the first run, and also to extend the measurements to both higher and lower pressures in a further attempt to observe the fourth structural phase predicted by Filinchuk et al. ${ }^{17}$ In this second cell we made a small modification of the wire probe to make it more rugged. Unfortunately, this modification also increased the errors due to end effects. Although the results for the two samples are in very good general agreement, the measured magnitude of $\kappa$ is about $2 \%$ higher for the second sample than for the first, and we believe that this difference is caused by the modification of the hot-wire layout.

In the course of this study we have collected a very large amount of data using three different methods. To present this information in a logical and structured way, we have chosen to discuss the properties of each structural phase and phase transformation in separate subsections below, starting with the phase stable under ambient conditions.

\section{A. Thermal properties of the tetragonal $14 \mathrm{~mm}$ phase}

The thermal conductivity of the orientationally disordered tetragonal $14 \mathrm{~mm}$ phase stable at near-ambient conditions is almost independent of $T$ but increases with increasing $p$. In the pressure range below $0.1 \mathrm{GPa}$ the $\mathrm{NH}_{3} \mathrm{BH}_{3}$ powder in the

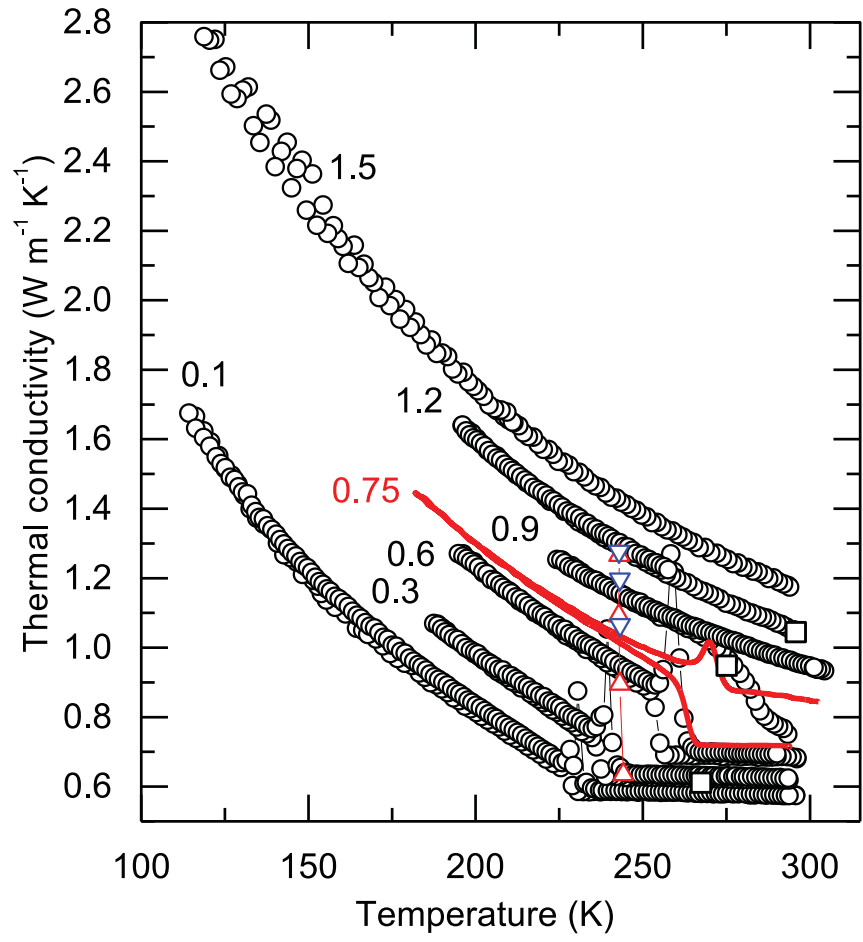

FIG. 1. (Color online) Thermal conductivity as a function of temperature at the pressures (in GPa) indicated. The anomalous data set at $0.75 \mathrm{GPa}$ is shown as a solid curve to make it easier for the reader to find(see text).

cell is consolidated and compressed into a nonporous solid by the applied load, and during this process the measured data for $\kappa$ are not reliable. Above $0.1 \mathrm{GPa}$, however, the data obtained are repeatable on pressure and temperature cycling for different samples, and we believe that they reflect the true bulk properties of $\mathrm{NH}_{3} \mathrm{BH}_{3}$.

The temperature dependence of $\kappa$ in the range 110 to $300 \mathrm{~K}$ at several pressures up to $1.5 \mathrm{GPa}$ is shown in Fig. 1. All data shown were obtained during isobaric cycles, starting at or near room temperature, then cooling to 110-220 K, after which the temperature was allowed to go back up to room temperature again. (In the run at $1.5 \mathrm{GPa}$ data are shown only for decreasing $T$; see Section $\mathrm{D}$ below.) It is clear from this figure that in the initial $I 4 \mathrm{~mm}$ phase $\kappa$ depends only weakly on $T$. Fitting a straight line to the data obtained at $0.1 \mathrm{GPa}$ we find $\kappa=0.656-2.7 \times 10^{-4} T \mathrm{~W} \mathrm{~m}^{-1} \mathrm{~K}^{-1}$, and a similar temperature dependence is observed in this phase at all pressures. A quite weak dependence on $T$ and $p$ is characteristic for the thermal conductivity of plastic crystal phases with rotational disorder, ${ }^{40}$ for example, solids based on quasi-spherical molecules such as ${ }^{41} \mathrm{CH}_{4}$ or ${ }^{39,42} \mathrm{C}_{60}$. However, even very long linear molecules such as $n$-alkanes up to $\mathrm{C}_{19} \mathrm{H}_{40}$ are observed to have plastic crystal phases with onedimensional molecular rotation and a low, almost temperatureindependent $\kappa$ at temperatures close to the melting point. ${ }^{43}$ The strong scattering of phonons by the rotational molecular motion causes an almost temperature-independent value for the phonon mean free path and thus also for $\kappa$, while the reduction in scattering by the combined effects of a slowing down of molecular rotation and a stiffening of the lattice due to 


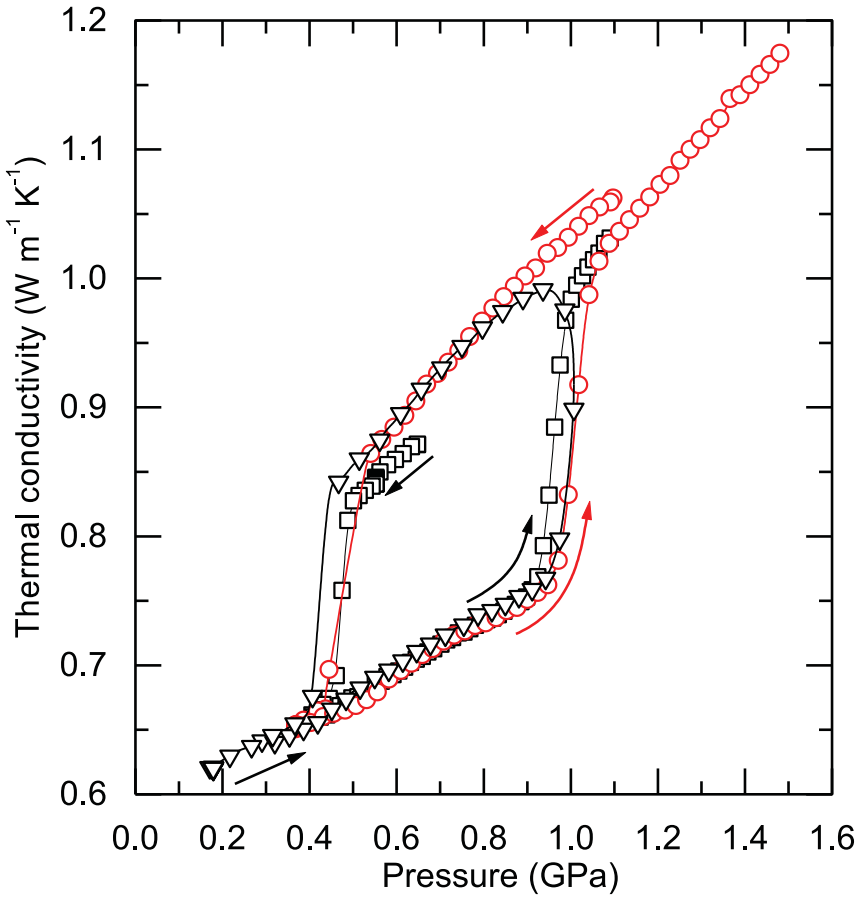

FIG. 2. (Color online) Thermal conductivity data from three pressure cycles at room temperature. Note the very large hysteresis of the $14 m m-C m c 2_{1}$ transformation.

increasing intermolecular interactions with increasing pressure allows a certain increase in $\kappa$ with $p$.

Data for $\kappa$ in the $14 \mathrm{~mm}$ phase are shown as functions of $p$ between 0.15 and $0.95 \mathrm{GPa}$ at room temperature in Fig. 2 (data at lower temperatures are shown in Fig. 4 below). To a good approximation $\kappa$ is a linear function of $p$, and using data from several pressure cycles we find an average pressure dependence $\kappa=0.563+0.195 p \mathrm{~W} \mathrm{~m}^{-1} \mathrm{~K}^{-1}$ between 0.2 and $0.9 \mathrm{GPa}$ at $293 \mathrm{~K}$. Extrapolating the data to atmospheric pressure we find an initial pressure coefficient of $d \ln \kappa / d p=$ $0.35 \mathrm{GPa}^{-1}$. To obtain the volume dependence we use the measured $^{17}$ zero-pressure bulk modulus $\mathrm{B}_{0}=9.9 \mathrm{GPa}$ to calculate the Bridgman coefficient $g=d \ln \kappa / d \ln V=$ 3.4. This rather low value falls well within the range of values observed for other plastic crystals. ${ }^{40}$ Similar results are found at lower temperatures.

In Table I we present measured numerical data for the thermal conductivity $\kappa$ in the tetragonal $14 \mathrm{~mm}$ phase, as well as for the other two phases investigated, at some selected $p-T$ coordinates.

\section{B. Properties of the low-temperature $P m n 2_{1}$ phase and the I4mm-Pmn2 $2_{1}$ transition}

In contrast to the behavior in the tetragonal phase, the thermal conductivity in the well-ordered orthorhombic lowtemperature $P m n 2_{1}$ phase shows a strong dependence on both $T$ and $p$ (see Fig. 1). At temperatures below the transition, a function

$$
k=A / T^{n}
$$

can be fitted to the data. For the $P m n 2_{1}$ phase the widest range in temperature, 110-225 K, was explored at 0.1 GPa. Fitting
TABLE I. Measured thermal conductivity $\left(\mathrm{W} \mathrm{m}^{-1} \mathrm{~K}^{-1}\right.$ ) of $\mathrm{NH}_{3} \mathrm{BH}_{3}$ at various pressures and temperatures. When possible, the values given are average values from more than one isobaric (or isothermal) experiment. Actual experimental values may differ depending on transition coordinates and thermal path followed by the sample. Some values have also been interpolated or extrapolated over small differences in $T$ and/or $p$.

\begin{tabular}{lllll}
\hline \hline GPA & \multicolumn{1}{c}{$293 \mathrm{~K}$} & \multicolumn{1}{c}{$250 \mathrm{~K}$} & \multicolumn{1}{c}{$200 \mathrm{~K}$} & $150 \mathrm{~K}$ \\
\hline 0.0 & $0.56^{\mathrm{a}}$ & $0.56^{\mathrm{a}}$ & $0.71^{\mathrm{b}, \mathrm{d}}, 0.95^{\mathrm{c}}$ & \\
0.1 & $0.58^{\mathrm{a}}$ & $0.59^{\mathrm{a}}$ & $0.83^{\mathrm{b}}, 1.06^{\mathrm{c}}$ & $1.22^{\mathrm{b}}$ \\
0.3 & $0.62^{\mathrm{a}}$ & $0.63^{\mathrm{a}}, 0.90^{\mathrm{c}}$ & $0.95^{\mathrm{b}}, 1.18^{\mathrm{c}}$ & \\
0.6 & $0.69^{\mathrm{a}}, 0.89^{\mathrm{c}}$ & $0.70^{\mathrm{a}}, 1.02^{\mathrm{c}}$ & $1.19^{\mathrm{b}}, 1.38^{\mathrm{c}}$ & \\
0.9 & $0.75^{\mathrm{a}}, 1.0^{\mathrm{c}}$ & $1.12^{\mathrm{c}}$ & $1.55^{\mathrm{b}}$ & \\
1.2 & $1.06^{\mathrm{c}}$ & $1.26^{\mathrm{c}}$ & $1.60^{\mathrm{c}}$ & $2.2^{\mathrm{c}, \mathrm{d}}$ \\
1.5 & $1.2^{\mathrm{c}}$ & $1.38^{\mathrm{c}}$ & $1.75^{\mathrm{c}}$ & $2.35^{\mathrm{c}}$ \\
\hline \hline
\end{tabular}

${ }^{\text {a In tetragonal } \mathrm{I} 4 \mathrm{~mm} \text { phase. }}$

${ }^{\mathrm{b}}$ In orthorhombic $P m n 2_{1}$ phase.

${ }^{\mathrm{c}}$ In orthorhombic $C m c 2_{1}$ phase.

${ }^{\mathrm{d}}$ Data extrapolated from higher pressure.

Eq. (1) to the data in this range gave $n=1.3$, and the fitted curve follows the data points quite closely except within about $20 \mathrm{~K}$ below the transition, where the measured data tend to deviate by curving downward. This might be connected with the strong precursor effects observed ${ }^{17,29,31}$ below the transition, which might either modify the phonon spectrum or increase scattering by the defects or disorder created, or both. For a normal, ordered crystal, where $\kappa$ is limited by phonon-phonon scattering, simple theory predicts $n=1$ at high temperatures (i.e., above the Debye temperature). The high value observed here for $n$ indicates that the somewhat brutal handling of the sample, involving severe plastic deformation during the compression of the original powder into a solid lump, still gives a very well-ordered crystalline material below the low-temperature phase transformation. Values higher than $n=1$ are not unusual and can be caused by several mechanisms. One possible reason might be that during cooling we approach the Umklapp freeze-out region, where $\kappa$ might even vary exponentially with temperature. However, a more likely explanation is that standard theory assumes constant volume conditions while our data were measured at constant pressure, a situation where thermal expansion effects are well known to result in a stronger temperature dependence than given by $n=1$. A third likely possibility is that the strong temperature dependence is caused by the rapid change in molecular motion with temperature ${ }^{44}$ (and pressure). Finally, it might be a consequence of the change in molecular orientation with temperature, ${ }^{17,31}$ but since the angle between the molecular and lattice axes changes by only a few degrees over a $100 \mathrm{~K}$ range we consider this less probable. To find the true cause for the effect, further calculations, preferably complemented by in situ NMR or neutron studies of the molecular dynamics, should be carried out for the $P m n 2_{1}$ structure under high-pressure conditions.

The pressure dependence of $\kappa$ was measured in several approximately isothermal pressure runs below $220 \mathrm{~K}$. It is clear from Fig. 1 that the pressure dependence in this phase is stronger than in the tetragonal phase. Fitting, as before, a straight line to data obtained between 0.3 and $0.7 \mathrm{GPa}$ at 
about $200 \mathrm{~K}$ we find $\kappa=0.712+0.71 p \mathrm{~W} \mathrm{~m}^{-1} \mathrm{~K}^{-1}$. Again extrapolating back to atmospheric pressure we find an initial pressure coefficient $d \ln \kappa / d p=1.0 \mathrm{GPa}^{-1}$ at $200 \mathrm{~K}$. We have not been able to find a reliable value for the bulk modulus of the $P m n 2_{1}$ phase, but if we assume an approximate value of $10 \mathrm{GPa}$, similar to those of the other two phases ${ }^{17}$ of $\mathrm{NH}_{3} \mathrm{BH}_{3}$, we find a Bridgman parameter $g=d \ln \kappa / d \ln V=10$. This value falls in the normal range of values found ${ }^{40}$ for other well-ordered, crystalline materials for which phonon-phonon scattering dominates $\kappa$.

Numerical data for $\kappa$ are again given in Table I. It should be noted that whenever possible the values given are average values from more than one isobaric or isothermal run. For the $P m n 2_{1}$ phase we also note that $\kappa$ depends surprisingly strongly on the thermal path followed in the experiment. At $200 \mathrm{~K}$, for example, $\kappa$ was up to $8 \%$ lower at any particular $p$ if the measurements were carried out under isothermal conditions (i.e., by changing the pressure) than when isobaric conditions (i.e., changing the temperature) were used. We have not been able to identify a clear reason for this interesting feature. One reasonable explanation is that isobaric temperature cycling is a rather "gentle" process, where the lattice may keep a well-ordered state throughout the cycle, resulting in a high $\kappa$. Isothermal pressure cycling, on the other hand, continuously deforms the lattice, breaking up crystallites and creating dislocations and defects, which produces a lower $\kappa$. Another possibility is that the changes in molecular orientation or rotation rate with pressure and temperature occur at different rates (or show hysteresis effects), such that the lattice structure may actually be slightly different at the same $p$ - $T$ coordinate depending on the thermal history.

Figure 1 shows that the transformation between the tetragonal $I 4 m m$ phase and the low-temperature $P m n 2_{1}$ phase changes character with pressure. At atmospheric pressure, this transition is close to second order with a very small volume change. ${ }^{17,30,31}$ The step change in $\kappa$ at $0.1 \mathrm{GPa}$ is also quite small, but with increasing $p$ the magnitude of the step increases such that at $0.6 \mathrm{GPa}$ it is about a factor of three larger. Although there could be many explanations for such an evolution, one possibility is that the step change in volume, and thus density, increases with increasing $p$ because the transformation changes to a character closer to first order. Stronger evidence for such an evolution can be found from data for the specific heat per unit volume, $\rho c_{p}$, shown in Fig. 3. Here $\rho$ is the density and $c_{p}$ is the specific heat per unit mass at constant pressure. Our measured data for $\rho c_{p}$ have a much larger inaccuracy than the data for $\kappa$, but relative values at different pressures and the general trend in the data should be reliable. Also, it should be taken into account that, in a dynamic method such as this, data are collected during a short temperature pulse with an amplitude of 2-4 K, and away from phase transformations the data for $\kappa$ and $c_{p}$ are thus average values over such a range in temperature. Within $2-4 \mathrm{~K}$ from a transformation, exothermal or endothermal effects also produce spurious responses such as the large peaks in $\kappa$ on heating, visible in the curves shown in Fig. 1 (caused by endothermal cooling simulating a very high $\kappa$ ). The data in Fig. 3, obtained during isobaric heating of the sample at different pressures, show that the pressure dependence of $\rho c_{p}$ is very small in both low-pressure phases. At temperatures

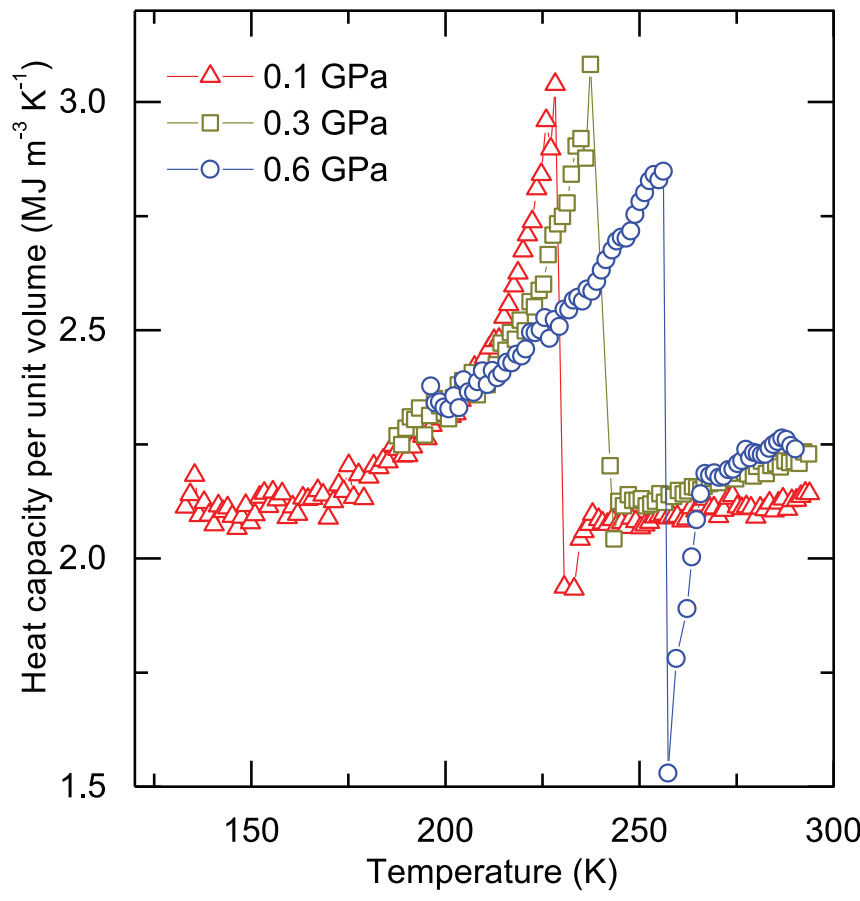

FIG. 3. (Color online) Measured data for the specific heat per unit volume as a function of temperature at $0.1,0.3$, and $0.6 \mathrm{GPa}$. The small rise below $150 \mathrm{~K}$ is an artifact of the method used.

below the transition, however, the data at $0.1 \mathrm{GPa}$ show a behavior which is quite close to the $\lambda$-type peak typical for second-order transformations, although the height and sharpness of the peak are blunted by the averaging effect inherent in the method. With increasing $p$ the second-order character visibly diminishes, as is also suggested by the data for $\kappa$. A change in the transition type under pressure is uncommon but not unique. A rather well-known example is the II-III transition in $\mathrm{NH}_{4} \mathrm{Cl}$, which goes the opposite way, from being first order at atmospheric pressure to becoming second order ${ }^{45-47}$ above about $0.2 \mathrm{GPa}$.

The rather large negative peak in $\rho c_{p}$ just above the transition at $0.6 \mathrm{GPa}$ is an unusual effect for which we have no clear explanation. It might be a dynamic effect, like the peaks in $\kappa$, but the fact that we do not usually observe such peaks suggests it might instead be connected with the formation of a small amount of a mixed orthorhombic phase, as will be discussed further below.

Finally, the $I 4 m m-P m n 2_{1}$ transformation shows only a small hysteresis, also in agreement with its weak first-order character. The small hysteresis is clearly visible in the isobaric temperature cycles shown in Fig. 1, and for completeness we show in Fig. 4 the results from two approximately isothermal pressure cycles through the phase line at temperatures just above $240 \mathrm{~K}$. Unfortunately, the temperature control is not perfect; note, however, that the "forward" $I 4 m m \rightarrow P m n 2_{1}$ transition in the first cycle (black circles) near $246 \mathrm{~K}$ occurs within $0.1 \mathrm{GPa}$ of the same pressure as the reverse transition in the second cycle (red squares) at $247 \mathrm{~K}$. The same goes for the transitions in the opposite directions, both occurring near $240 \mathrm{~K}$. 


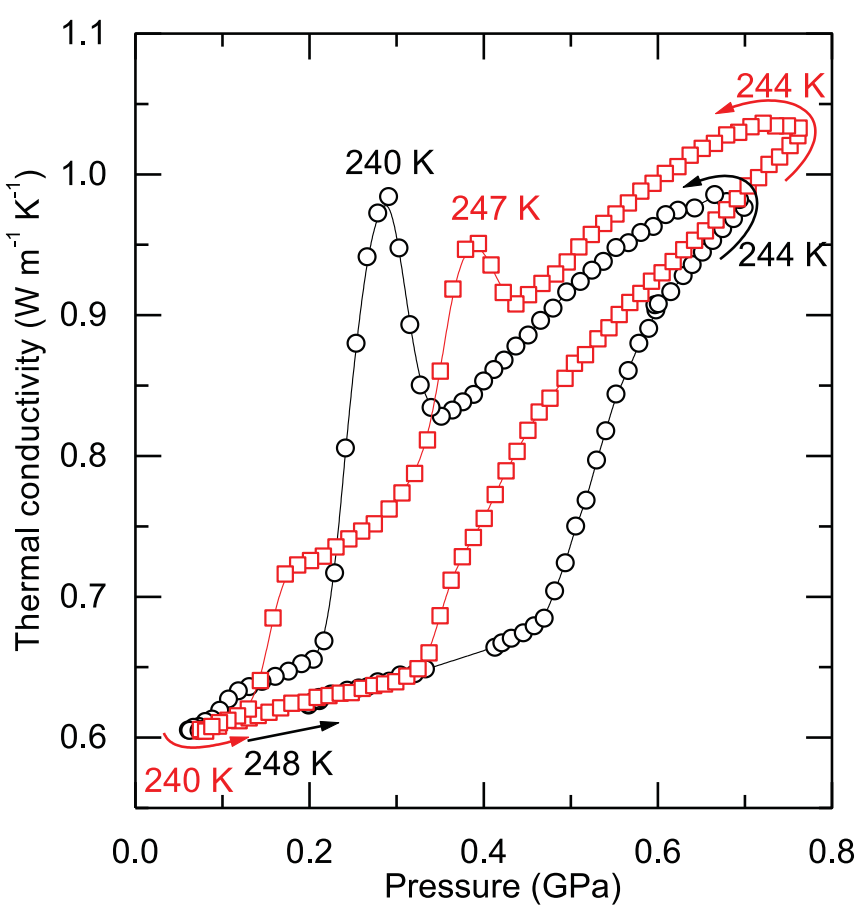

FIG. 4. (Color online) Thermal conductivity as a function of pressure, measured during two pressure cycles near $245 \mathrm{~K}$. Transformations between the low-pressure tetragonal $14 \mathrm{~mm}$ phase and the orthorhombic Pmn2 $2_{1}$ phase are clearly seen (see text for details).

\section{Properties of the high-pressure $C m c 2_{1}$ phase and the I4mm-Cmc2 $2_{1}$ transformation behavior}

On increasing $p$ to above $0.9 \mathrm{GPa}$ at room temperature we observed a phase transformation which we identified as the well-known transition ${ }^{13-21}$ from the tetragonal structure into the known ${ }^{17,20,21}$ orthorhombic $C m c 2_{1}$ phase. The step change in $\kappa$ at this transition was similar to, or larger than, that observed for the $I 4 m m-P m n 2_{1}$ transition at $0.6 \mathrm{GPa}$. As shown in Fig. 2, the $14 m m-C m c 2_{1}$ transformation was also reversible, but the reverse transformation back to the tetragonal phase did not occur until $p$ was decreased to well below $0.6 \mathrm{GPa}$. A cooling experiment carried out at $0.9 \mathrm{GPa}$ (Fig. 1) showed a rather sluggish transformation, again involving a total change in $\kappa$ of about the same magnitude, and on reheating to room temperature we could not observe any reverse transformation until the pressure was again decreased by a large amount. In Fig. 1 we also show a cooling cycle at $1.2 \mathrm{GPa}$ and a cooling curve at $1.5 \mathrm{GPa}$, neither of which shows any transformations since we start with the sample in the $C m c 2_{1}$ phase.

The temperature dependence of $\kappa$ in the $C m c 2_{1}$ phase is well described by Eq. (1) with $n \approx 1.0$ at 1.2 and $1.5 \mathrm{GPa}$, showing that we have another well-crystallized material with $\kappa$ limited primarily by phonon-phonon interactions. Figures 1 and 2 show that the pressure dependence is again stronger than in the tetragonal low-pressure phase. A linear fit to the data between 1.1 and $1.5 \mathrm{GPa}$ yields $\kappa=0.619+$ $0.38 p \mathrm{~W} \mathrm{~m} \mathrm{~m}^{-1} \mathrm{~K}^{-1}$ at room temperature, from which we calculate a pressure coefficient $\mathrm{d} \ln \kappa / \mathrm{d} p=0.37 \mathrm{GPa}^{-1}$ at $1.1 \mathrm{GPa}$. Using the room-temperature value for the bulk modulus $^{17}$ at this pressure, $B(1.1 \mathrm{GPa})=14.9 \mathrm{GPa}$, we find a Bridgman parameter $g=d \ln \kappa / d \ln V=5.5$, significantly lower than for the orthorhombic $P m n 2_{1}$ phase but still in the range expected for well ordered crystals. ${ }^{40} \mathrm{~A}$ rather uncertain extrapolation to atmospheric pressure, carried out for comparison purposes, gives a slightly higher $g \approx 6.2$. Numerical data for the thermal conductivity can again be found in Table I.

For the first sample we mainly obtained data for the hysteresis near room temperature, but for the second sample we carried out a large number of pressure and temperature cycles. The $C m c 2_{1}$ phase was usually produced near $1 \mathrm{GPa}$ at room temperature, after which the sample was cooled at constant pressure to some lower temperature. The tetragonal $14 \mathrm{~mm}$ phase was then recovered by crossing the stability boundary either by decreasing the pressure at low $T$ along an isothermal path, or by first reducing the pressure, then heating the sample at constant $p$. The transformation from the tetragonal phase into the $C m c 2_{1}$ phase always occurred on a phase line connecting the known room-temperature transition point at $0.9-1.0 \mathrm{GPa}$ with the phase line of the reversible $I 4 m m-P m n 2_{1}$ phase line near $0.7 \mathrm{GPa}$. The slope of this line is about $140 \mathrm{~K} \mathrm{GPa}^{-1}$. On the other hand, in both isobaric and isothermal experiments the reverse transformations into tetragonal $\mathrm{NH}_{3} \mathrm{BH}_{3}$ were found to outline a single, well-defined line of stability running from about $0.52 \mathrm{GPa}$ near $300 \mathrm{~K}$ to $232 \mathrm{~K}$ at atmospheric pressure and with a slope increasing from about $85 \mathrm{~K} \mathrm{GPa}^{-1}$ near atmospheric pressure to $170 \mathrm{~K} \mathrm{GPa}^{-1}$ at $300 \mathrm{~K}$. The resulting phase diagram of $\mathrm{NH}_{3} \mathrm{BH}_{3}$ is shown in Fig. 5.

We note that Chen et al. ${ }^{21}$ very recently found a negative slope $d T / d p$ for the $I 4 m m$ to $C m c 2_{1}$ transition line above room temperature, and concluded that the transition was

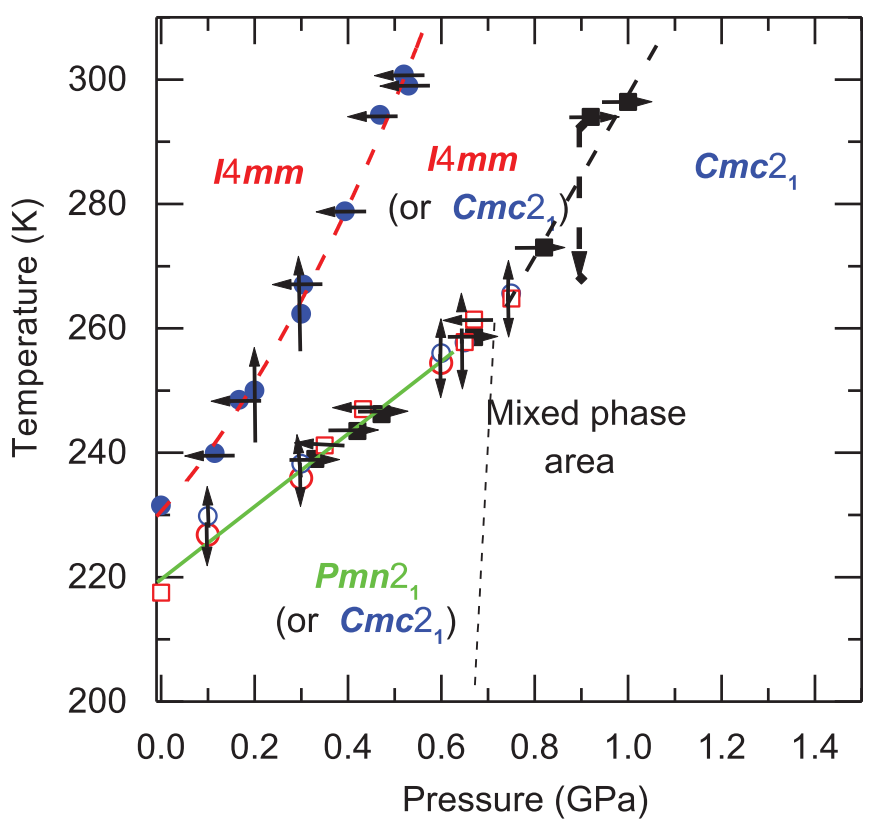

FIG. 5. (Color online) Pressure-temperature phase diagram of $\mathrm{NH}_{3} \mathrm{BH}_{3}$ derived in the present paper. Symbols show coordinates of the transformations observed, and arrows show both the transformation direction and the approximate transition ranges observed. Full (green) line denotes the $I 4 m m \leftrightarrow P m n 2_{1}$ transition, dashed lines transformations between $I 4 \mathrm{~mm}$ and $C m c 2_{1}$ phases. Dotted, almostvertical line denotes approximate locations for the $P m n 2_{1} \rightarrow C m c 2_{1}$ transformation. 
endothermal. In our experiments, direct in situ measurements of the sample temperature using a thermocouple show instead large exothermal anomalies at the transition, supporting the fact that the measured phase line slopes are positive for both transition directions at and below room temperature.

The stability of the high-pressure $C m c 2_{1}$ phase was also studied by Raman spectroscopy in a separate set of experiments. The sample was pressurized to $1.4 \mathrm{GPa}$ at $293 \mathrm{~K}$ to ensure complete transformation into the $C m c 2_{1}$ phase, and $p$ was then decreased in three steps to $1.1,1.0$, and $0.85 \mathrm{GPa}$ with cooling runs down to $\sim 230-240 \mathrm{~K}$ at every step. In all three cooling experiments the pure $C m c 2_{1}$ phase was preserved to the lowest temperatures, and no phase transitions were observed. Further Raman studies will be presented in the next section.

It is clear that the transformation behaviors of the two orthorhombic phases are quite different, with a very much larger hysteresis for the high-pressure $C m c 2_{1}$ phase, possibly because the deformation of the high-pressure $C m c 2_{1}$ phase with respect to the parent $I 4 \mathrm{~mm}$ structure is much larger than for the low-temperature $P m n 2_{1}$ phase. ${ }^{17}$ It has also been shown ${ }^{17}$ that the patterns of dihydrogen bonds are very similar in the ambient-pressure phases, while in the high-pressure phase the large tilts of the $\mathrm{NH}_{3} \mathrm{BH}_{3}$ molecules with respect to the $c$ axis result in a different and more complex pattern of H. ..H interactions. Once formed, the $C m c 2_{1}$ phase is very stable, and at $0.2 \mathrm{GPa}$ and higher pressures our thermal conductivity measurements do not show indications of any transformation from this phase into the low-temperature orthorhombic $P m n 2_{1}$ structure. In one of our final experiments, however, we brought a $C m c 2_{1}$ sample all the way down to atmospheric pressure, at which we heated it back up to room temperature. As shown in Fig. 6, we could then clearly see two transitions, one at $218 \mathrm{~K}$, in excellent agreement with the $P m n 2_{1}$ to $I 4 m m$ transition point observed by Filinchuk et al. ${ }^{17}$ and one at $232 \mathrm{~K}$, corresponding to the $C m c 2_{1}$ to $I 4 m m$ transition. The $C m c 2_{1}$ phase thus seems to be at least partially stable even at atmospheric pressure, and then at temperatures even higher than for the normal low-pressure orthorhombic Pmn2 1 phase.

\section{Mixed-phase region, $P m n 2_{1}$-to- $C m c 2_{1}$ transformations, and the search for a new phase}

As mentioned above, the thermal conductivity measurements on the first sample showed anomalous values for $\kappa$ near $0.7 \mathrm{GPa}$. To investigate whether we had found the fourth phase predicted by Filinchuk et $a{ }^{17}{ }^{17}$ we therefore carried out a series of in situ Raman investigations during isobaric cooling-heating cycles at temperatures down to 240-250 K and with initial pressures of $0.8,0.9,1.0$ (Fig. 7), and $1.1 \mathrm{GPa}$. The results obtained showed clear, reversible, systematic changes in the Raman spectra with $T$ in the range just below the observed tetragonal-orthorhombic transition boundary. In Fig. 8 we compare experimental Raman spectra measured for the three known pure phases with a typical spectrum as obtained in the range $0.8-1 \mathrm{GPa}$ below $280 \mathrm{~K}$. The new spectra basically contain features characteristic for both known orthorhombic phases, and a preliminary conclusion must be that the data collected near the phase boundary are

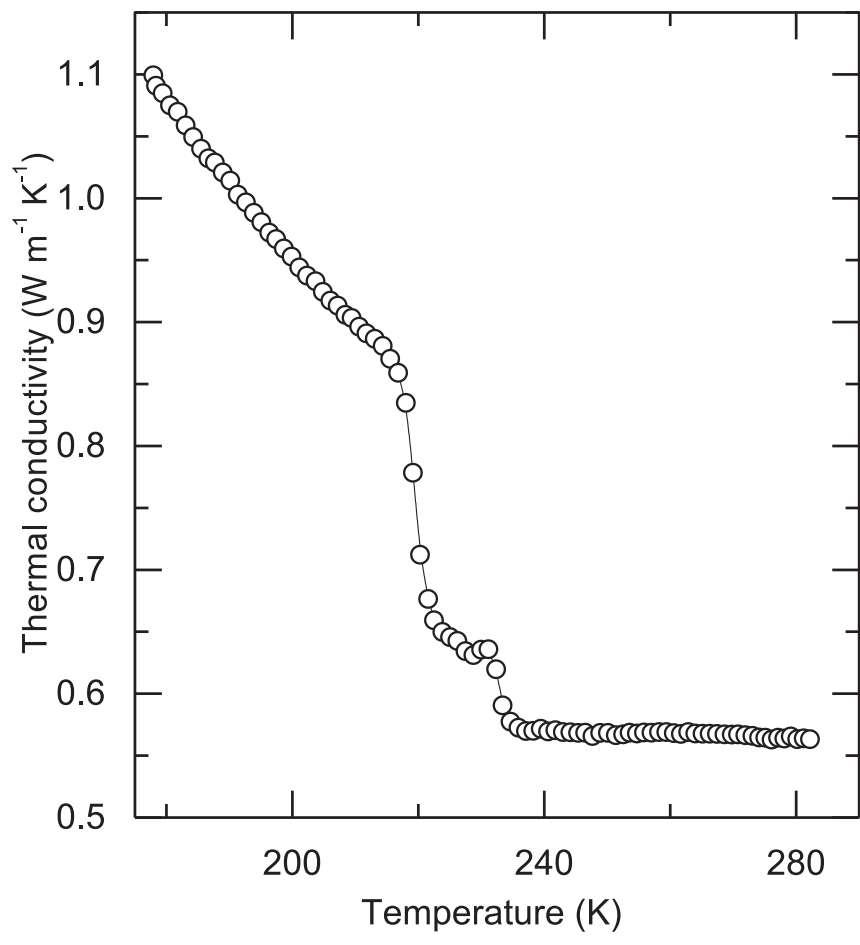

FIG. 6. Thermal conductivity as a function of temperature, measured during heating at atmospheric pressure after producing the orthorhombic $C m c 2_{1}$ phase at $1 \mathrm{GPa}$ at room temperature.

compatible with mixtures of the known phases. However, because the Raman spectra of the various phases of $\mathrm{NH}_{3} \mathrm{BH}_{3}$ are very similar, the existence of a new phase could not be completely ruled out.

To find the exact phase composition of $\mathrm{NH}_{3} \mathrm{BH}_{3}$ in the low-temperature $(230-250 \mathrm{~K})$, high-pressure $(0.7-1.0 \mathrm{GPa})$ regime, we therefore carried out a set of XRD experiments. The experiments started at $0.8 \mathrm{GPa}$ and room temperature, and

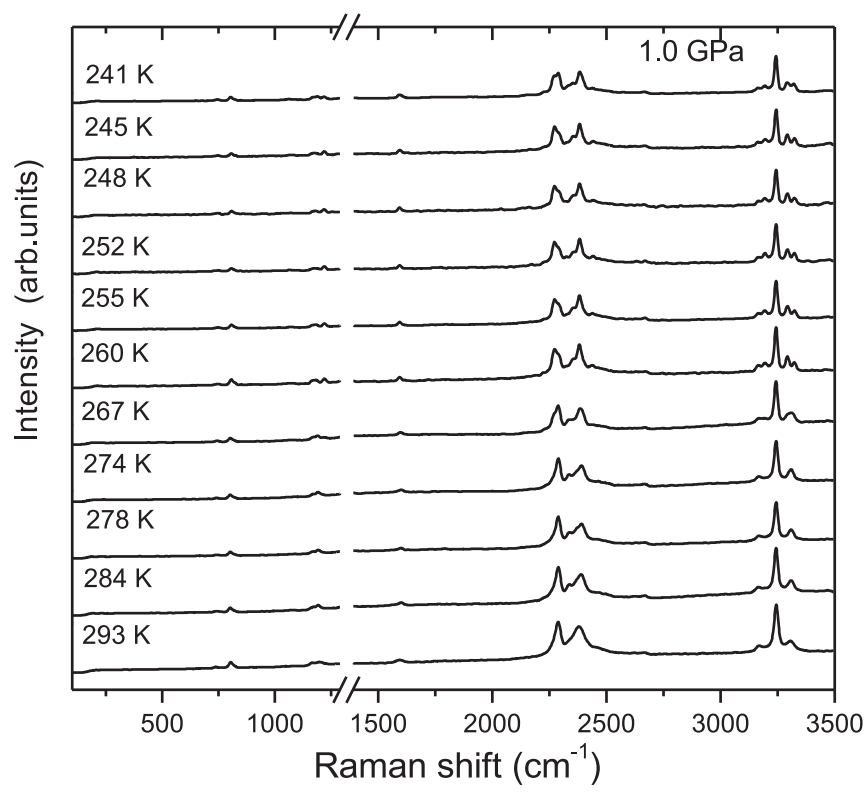

FIG. 7. Raman spectra recorded from $\mathrm{NH}_{3} \mathrm{BH}_{3}$ upon cooling at $1 \mathrm{GPa}$. 


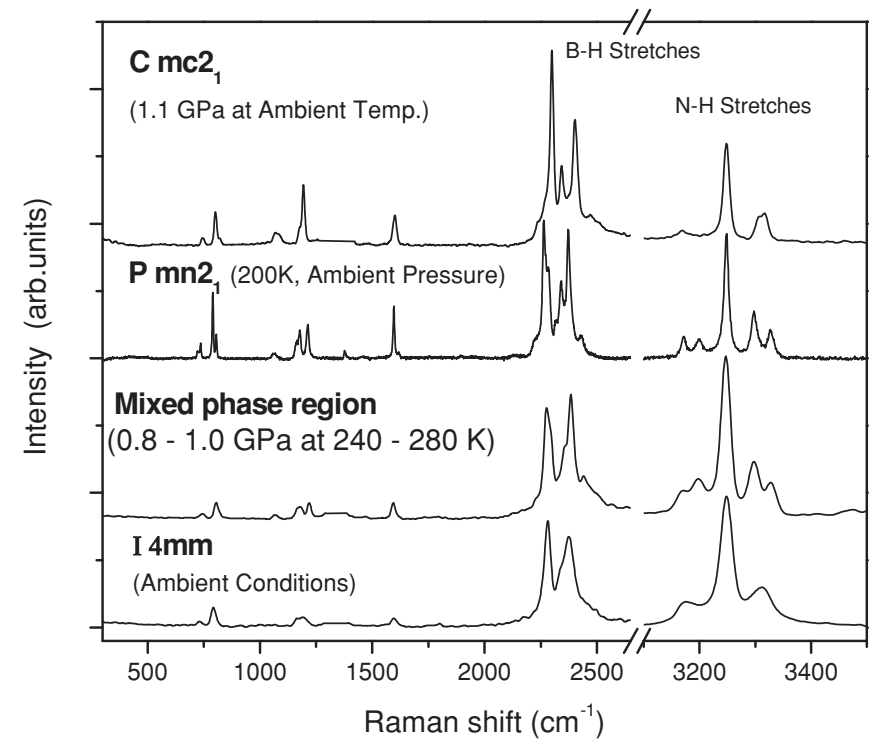

FIG. 8. Comparison between Raman spectra of the known phases of $\mathrm{NH}_{3} \mathrm{BH}_{3}$ and the mixed phase obtained below $280 \mathrm{~K}$ at intermediate pressures.

the samples were cooled at constant $p$ down to $220 \mathrm{~K}$. Below $259 \mathrm{~K}$ a clear phase transition was observed (Fig. 9), and a detailed analysis of the diffraction data proved that this phase transition does not involve any new, unknown phase. Rietveld analysis of the pattern recorded at $220 \mathrm{~K}$ (Fig. 10) showed that the sample consisted of the two known orthorhombic phases, $C m c 2_{1}(a=5.909 \AA, b=6.413 \AA, c=6.052 \AA)$ and $P m n 2_{1}$ $(a=5.419 \AA, b=4.494 \AA, c=4.843 \AA)$. The complete refinement also included two weak lines from the gasket; these are not shown in the figure to avoid confusing the reader. The relative amounts of these two phases change upon cooling, as can be seen in Fig. 9: The reflections from the $C m c 2_{1}$ phase

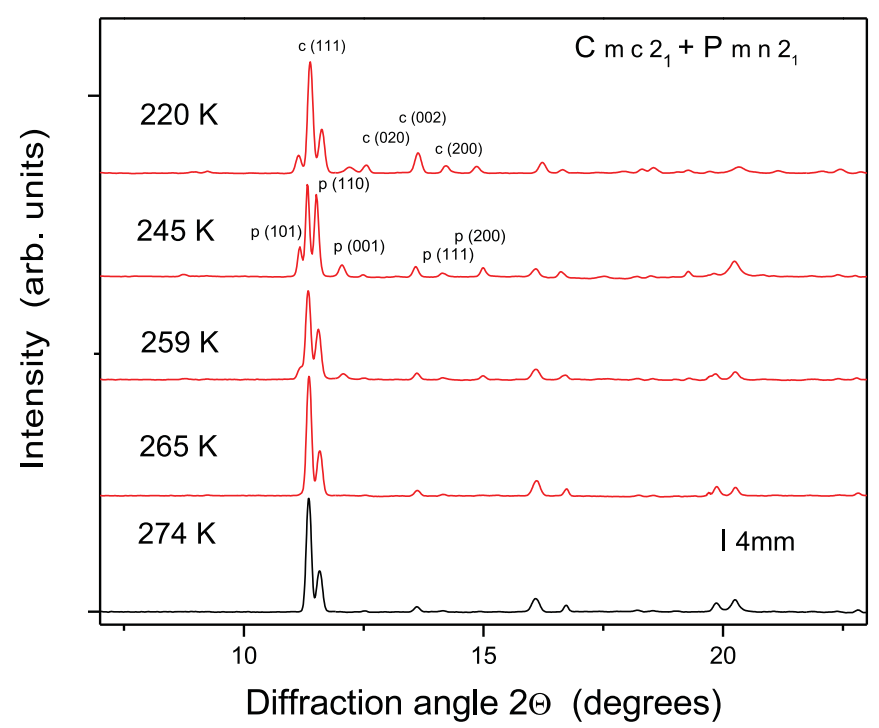

FIG. 9. XRD patterns recorded from $\mathrm{NH}_{3} \mathrm{BH}_{3}$ upon cooling at $0.8 \mathrm{GPa}$. Indexing for some reflections from the $P m n 2_{1}$ phase (p) is shown on the $245 \mathrm{~K}$ pattern and for the $C m c 2_{1}$ phase on the $220 \mathrm{~K}$ pattern.

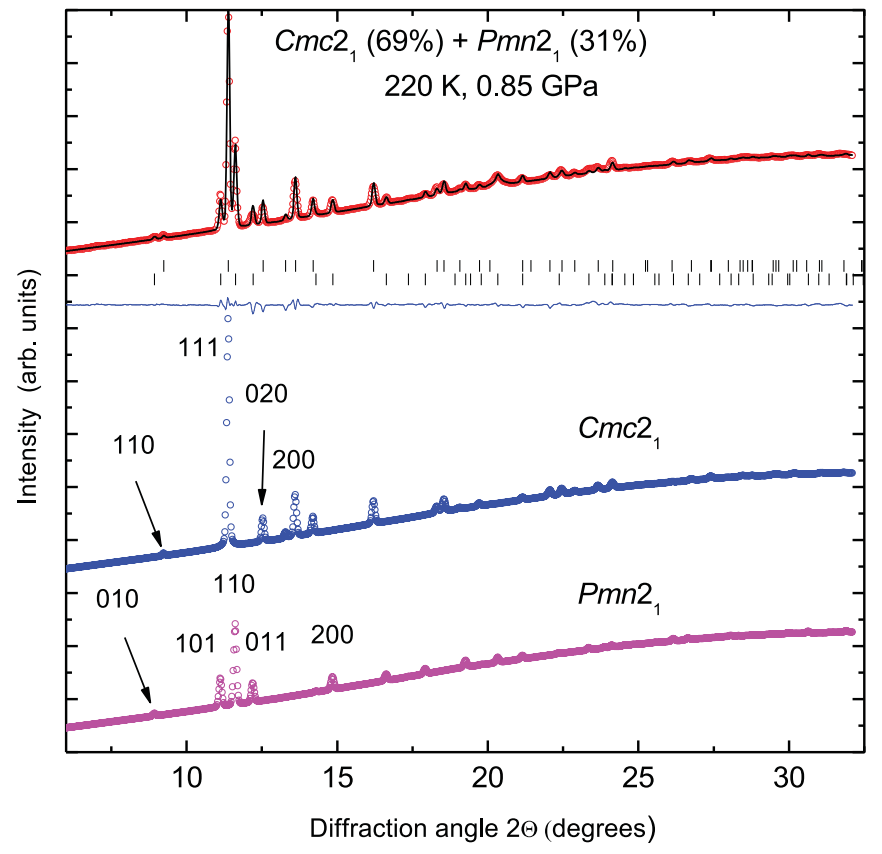

FIG. 10. (Color online) Rietveld refinement profile for the mixed orthorhombic phase (top) together with the contributions from the separate phases (bottom two curves). Tics corresponding to peak positions for the two phases (Cmc2 $2_{1}$ top, $P m n 2_{1}$ below) are shown together with the residual difference below the refinement profile (for fitted lattice parameters, see text).

become significantly stronger at lower temperatures while at $245 \mathrm{~K}$ the majority phase is $P m n 2_{1}$.

After this final identification of the structure, we investigated the behavior in the "mixed-phase" pressure range by further measurements of $\kappa$ for the second sample. Figure 11 shows results obtained during repeated cycling through the tetragonal-orthorhombic phase line at $0.65 \mathrm{GPa}$, close to the point where the phase line changes its slope. During the first cooling run a step increase in $\kappa$ indicates that a transition is taking place, while on heating back through the phase line the value of $\kappa$ decreases only halfway toward its initial value. This behavior is very similar to that found earlier at $0.75 \mathrm{GPa}$ (Fig. 1). On repeated cycling, the high-temperature value for $\kappa$ becomes increasingly higher for each cycle. We interpret this as the creation of a continuously increasing fraction of the $C m c 2_{1}$ phase. Each time we cool through the phase line the tetragonal phase present transforms into a mixture of the two orthorhombic phases. On heating, however, only the $P m n 2_{1}$ phase reverts to the tetragonal phase, while the $C m c 2_{1}$ phase remains in its metastable orthorhombic state. This leads to a continuously increasing fraction of the latter phase in the sample on temperature cycling. We note that $\kappa$ increases slightly with cycling also in the low-temperature state, as the fraction of the $C m c 2_{1}$ phase approaches 1 and the overall crystalline order increases. However, the existence of a transformation peak shows that small amounts of the $P m n 2_{1}$ phase are still formed from residual tetragonal $\mathrm{NH}_{3} \mathrm{BH}_{3}$ even in the third cycle, although this does not show up in the measured magnitude of $\kappa$.

Because the magnitudes of $\kappa$ are very similar in the two orthorhombic phases (see Table I) it was difficult to 


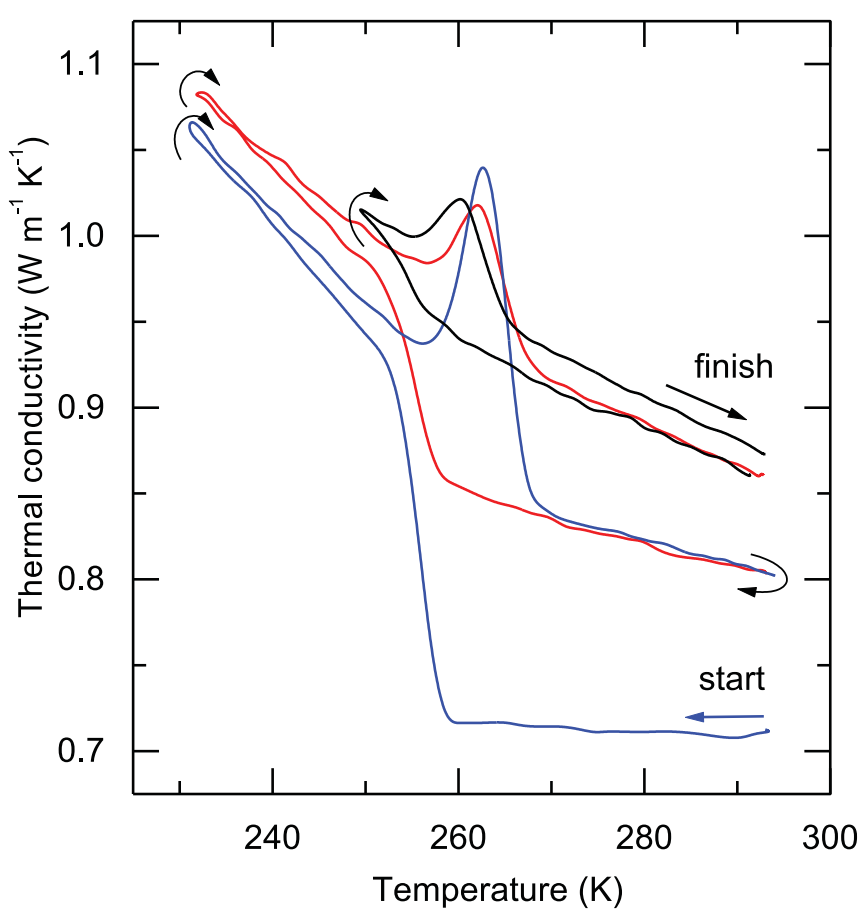

FIG. 11. (Color online) Thermal conductivity data measured during three temperature cycles at $0.65 \mathrm{GPa}$. See text for details.

observe transformations between these phases by thermal measurements, and for the first sample we carried out both an isothermal cycle up to $1.2 \mathrm{GPa}$ at $200 \mathrm{~K}$ and an isobaric cycle down to $200 \mathrm{~K}$ at $1.2 \mathrm{GPa}$ without observing any phase transitions. As discussed above, the $C m c 2_{1}$ phase is very stable and we have not observed any transformation of this phase into $P m n 2_{1}$ at any pressure in the range $p \geqslant 0.2 \mathrm{GPa}$. The reverse transition, however, could be observed. In the measurements of $\kappa$, the transition was observed only indirectly while pressure cycling at low temperatures. Figure 4, which was already discussed above, shows data for $\kappa$ as a function of $p$ near $245 \mathrm{~K}$ measured during two pressure cycles, one up to $0.65 \mathrm{GPa}$ and the other to $0.7 \mathrm{GPa}$. When decreasing $p$ in the first case (black circles), the $P m n 2_{1}$ to $I 4 m m$ transition is observed at the pressure expected from the (reversible) phase line in Fig. 5 (full green line). When the experiment is repeated (red squares), extending the pressure cycle to slightly higher pressure, we observe the same reverse transformation, but even after this transition part of the sample obviously remains orthorhombic and transforms only at the stability boundary for the $C m c 2_{1}$ phase near $0.15 \mathrm{GPa}$. A small fraction of the sample thus transformed from $P m n 2_{1}$ to $C m c 2_{1}$ between 0.65 and $0.7 \mathrm{GPa}$. In fact, a very small "foot" observed in the first curve at the same low pressure indicates that a small amount of the $C m c 2_{1}$ phase is also formed during the first pressure cycle to $0.65 \mathrm{GPa}$. In another pressure run, between 0.3 and $1.1 \mathrm{GPa}$ near $200 \mathrm{~K}$, we also observe a change in the slope $\mathrm{d} \kappa / \mathrm{d} p$ by about $25 \%$ just below $0.7 \mathrm{GPa}$, which might indicate the same (slow) transition. However, in view of the actual temperature stability during the run and the large temperature dependence of $\kappa$ in this range of temperature we cannot use this change as conclusive evidence.

Direct evidence for such a transition could, however, be found in Raman measurements. To observe the transition, we carried out an experiment starting at $1 \mathrm{GPa}$ and room temperature. The sample was cooled to $239 \mathrm{~K}$, during which the phase transition into the mixed phase was clearly observed. The pressure was then increased isothermally to $\sim 1.4 \mathrm{GPa}$ at low $T$, without any observable change in the Raman spectrum, after which the sample was heated back to room temperature. During this last heating at $1.4 \mathrm{GPa}$ the phase transition into pure $C m c 2_{1}$ was observed at about $250 \mathrm{~K}$, where all spectral features of the $P m n 2_{1}$ disappeared.

The three different methods thus give somewhat different results for the $P m n 2_{1}$-to- $C m c 2_{1}$ transformation. The measurements of $\kappa$ show that a slow transformation occurs at low temperatures at pressures above $0.65 \mathrm{GPa}$; the Raman data show that the transformation occurs mainly on heating to above $250 \mathrm{~K}$ at high pressures $(1.4 \mathrm{GPa})$, while the XRD study shows that the fraction of the $C m c 2_{1}$ phase increases steadily with decreasing $T$ during cooling at $0.8 \mathrm{GPa}$. We conclude that there seems to be a stability limit near 0.6-0.7 GPa above which the $P m n 2_{1}$ phase slowly transforms into the $C m c 2_{1}$ structure and that the actual structure in this area depends on the thermal history of the sample in a complicated way.

To finally verify the phase diagram in Fig. 5, we carried out measurements of $\kappa$ around closed loops in the $p$ - $T$ plane. Starting at $0.3 \mathrm{GPa}$ at room temperature we increased $p$ to $1.2 \mathrm{GPa}$, then cooled to $210 \mathrm{~K}$, decreased $p$ to $0.3 \mathrm{GPa}$, and heated back to our original starting coordinates. This cycle was then reversed, following (approximately) the same trajectory in the reverse direction back to the origin. During the clockwise cycle, only transitions identified as transformations between the $I 4 \mathrm{~mm}$ and $C m c 2_{1}$ phases were observed, as expected from the metastability properties of the latter phase. During the anticlockwise cycle, the only transitions observed occurred at the $I 4 m m-P m n 2_{1}$ phase line and at the $C m c 2_{1} \rightarrow I 4 m m$ phase line. However, as mentioned above we also observed a small change of slope $d \kappa / d p$ near $0.7 \mathrm{GPa}$ at $200 \mathrm{~K}$. From the data shown in Fig. 4, the Raman data referred to above, and the discussion above we conclude that a transformation from $P m n 2_{1}$ to $C m c 2_{1}$ may have occurred somewhere near or above $0.7 \mathrm{GPa}$ at $210 \mathrm{~K}$, possibly resulting in the observed change in $d \kappa / d p$. However, all or part of the transformation may also have occurred during heating to room temperature at 1.2 GPa. It seems that the transformation is too slow and gradual to be clearly identified by the methods used under these $p$ - $T$ conditions. We have thus not been able to map this transition boundary in detail, and we have only tentatively indicated a possible location for the corresponding phase boundary by a dotted line in the phase diagram of Fig. 5 . The slope of this line is shown as positive, because the XRD data show an increasing fraction of the $C m c 2_{1}$ phase on cooling. The kinetics and details of this transition have not been identified.

In several of the studies discussed in this and earlier sections, a secondary aim was to find and identify the fourth structural phase predicted by Filinchuk et al., ${ }^{17}$ as discussed above. This phase should appear at low temperatures, possibly in a $p-T$ area between the ranges of stability of the two orthorhombic phases, i.e., in the mixed-phase area or near the dotted line shown in the phase diagram (Fig. 5). In addition to the many isobaric and isothermal cycles carried out in this $p-T$ range we also finished the 
low-temperature run at $1.5 \mathrm{GPa}$ (Fig. 1) by continuously decreasing $p$ during the final heating, thus passing "diagonally" across the region of interest at temperatures well below $200 \mathrm{~K}$. Even in this case the data for $\kappa$ still showed no indications of a further transformation, and we must conclude that in the range up to $1.5 \mathrm{GPa}$ we do not see any indications of such a new phase at any temperature above $110 \mathrm{~K}$.

\section{CONCLUSIONS}

In this paper we have carried out a detailed investigation of the $p-T$ phase diagram of $\mathrm{NH}_{3} \mathrm{BH}_{3}$, including hysteresis effects, in the range $110-300 \mathrm{~K}$ and $0-1.5 \mathrm{GPa}$. We find the known transformations from the parent tetragonal structure into the two orthorhombic low-temperature and high-pressure structures at temperatures and pressures in excellent agreement with literature data, and we have been able to map the phase boundaries connecting these points with high accuracy, as shown in Fig. 5 above. However, in the $p-T$ range investigated we have found no evidence for the fourth phase predicted by Filinchuk et al., ${ }^{17}$ and the search for this phase should thus be continued at higher pressures and/or lower temperatures.

The transformation between the tetragonal phase and the low-temperature $P m n 2_{1}$ phase is always reversible and associated with a quite small hysteresis. Data for both the thermal conductivity and the specific heat capacity indicate an evolution of the transformation with pressure from the almost second-order transition observed ${ }^{17,30,31}$ near atmospheric pressure toward a stronger first-order character near $0.5 \mathrm{GPa}$, but the slope of the associated phase line still seems to be almost constant near $60 \mathrm{~K} \mathrm{GPa}^{-1}$. We find that the orthorhombic $P m n 2_{1}$ phase transforms into the likewise orthorhombic $\mathrm{Cmc} 2_{1}$ phase at pressures above $0.7 \mathrm{GPa}$, but different methods have given very different results for the transformation coordinates and we have not been able to identify a unique transition boundary, probably because the kinetics are slow and the transformation occurs gradually.

The phase boundary for the tetragonal-to-orthorhombic $C m c 2_{1}$ transition connects the end of the $I 4 m m-P m n 2_{1}$ phase line with the known transition point near $1 \mathrm{GPa}$ at room temperature and has an approximate slope of $140 \mathrm{~K} \mathrm{GPa}^{-1}$. Direct in situ thermal measurements show that the transition is exothermal, in contradiction to a recently published result. ${ }^{21}$ The $C m c 2_{1}$ phase is highly metastable. In our experiments it transforms partially into the $P m n 2_{1}$ phase at low temperature, but only below $0.2 \mathrm{GPa}$, and the transformation back to the tetragonal phase occurs at pressures very much lower (temperatures much higher) than the forward transformation. The transformation points define a slightly curving phase boundary starting just above $230 \mathrm{~K}$ at atmospheric pressure and having a high-temperature slope near $170 \mathrm{~K} \mathrm{GPa}^{-1}$. The typical hysteresis of this transition is about $0.5 \mathrm{GPa}$. The (meta)stability of the high-pressure phase may originate from a very different pattern of the dihydrogen $\mathrm{H}^{\delta+} \cdots \mathrm{H}^{\delta-}$ interactions compared with the ones in the ambient-pressure phases. ${ }^{17}$ The strong metastability of the orthorhombic $C m c 2_{1}$ phase indicates that it might be possible to stabilize denser lattice structures, with higher volumetric hydrogen densities than the normal zero-pressure phases, in the $\mathrm{NH}_{3} \mathrm{BH}_{3}$ system by doping the material with impurities or by other means.

\section{ACKNOWLEDGMENTS}

This work was financially supported by Carl Tryggers Stiftelse för Vetenskaplig Forskning and by the Swiss-Norwegian Beam Lines at ESRF through the allocation of in-house beam time. The authors wish to thank Dmitry Chernyshov for his help with the diffraction experiments. *bertil.sundqvist@physics.umu.se

${ }^{1}$ L. Schlapbach and A. Züttel, Nature (London) 414, 353 (2001).

${ }^{2}$ Y. Filinchuk, D. Chernyshov, and V. Dmitriev, Z. Kristallogr. 223, 649 (2008).

${ }^{3}$ B. Sundqvist, Solid State Phenom. 150, 175 (2009), [http://www.scientific.net/SSP.150.175].

${ }^{4}$ B. Sundqvist and O. Andersson, Int. J. Thermophys. 30, 1118 (2009).

${ }^{5}$ L. George and S. K. Saxena, In. J. Hydrogen Energy 35, 5454 (2010).

${ }^{6}$ B. C. Hauback, Z. Kristallogr. 223, 636 (2008).

${ }^{7}$ A. Staubitz, A. P. M. Robertson, and I. Manners, Chem. Rev. 110, 4079 (2010).

${ }^{8}$ M. G. Hu, R. A. Geanangel, and W. W. Wendlandt, Thermochim. Acta 23, 249 (1978).

${ }^{9}$ F. H. Stephens, V. Pons, and R. T. Baker, Dalton Trans. 2613 (2007).

${ }^{10}$ Z. T. Xiong, C. K. Yong, G. T. Wu, P. Chen, W. Shaw, A. Karkamkar, T. Autrey, M. O. Jones, S. R. Johnson, P. P. Edwards, and W. I. F. David, Nat. Mater. 7, 138 (2008).

${ }^{11}$ H. Wu, W. Zhou, F. E. Pinkerton, M. S. Meyer, G. Srinivas, T. Yildirim, T. J. Udovic, and J. J. Rush, J. Mater. Chem. 20, 6550 (2010).
${ }^{12}$ W. Li, R. H. Scheicher, C. M. Araújo, G. T. Wu, A. Blomqvist, C. Z. Wu, R. Ahuja, Y. P. Feng, and P. Chen, J. Phys. Chem. C 114, 19089 (2010).

${ }^{13}$ S. Trudel and D. F. R. Gilson, Inorg. Chem. 42, 2814 (2003).

${ }^{14}$ R. Custelcean and Z. A. Dreger, J. Phys. Chem. B 107, 9231 (2003).

${ }^{15}$ Y. Lin, W. L. Mao, V. Drozd, J. H. Chen, and L. L. Daemen, J. Chem. Phys. 129, 234509 (2008).

${ }^{16}$ R. S. Chellappa, M. Somayazulu, V. V. Struzhkin, T. Autrey, and R.S. Hemley, J. Chem. Phys. 131, 224515 (2009).

${ }^{17}$ Y. Filinchuk, A. H. Nevidomskyy, D. Chernyshov, and V. Dmitriev, Phys. Rev. B 79, 214111 (2009).

${ }^{18}$ S.T. Xie, Y. Song, and Z. X. Liu, Can. J. Chem. 87, 1235 (2009).

${ }^{19}$ J. Nylén, T. Sato, E. Soignard, J. L. Yarger, E. Stoyanov, and U. Häussermann, J. Chem. Phys. 131, 104506 (2009).

${ }^{20}$ R. S. Kumar, X. Z. Ke, J. Z. Zhang, Z. J. Lin, S. C. Vogel, M. Hartl, S. Sinogeikin, L. Daemen, A. L. Cornelius, C. F. Chen, and Y. S. Zhao, Chem. Phys. Lett. 495, 203 (2010).

${ }^{21}$ J. H. Chen, H. Couvy, H. Z. Liu, V. Drozd, L. L. Daemen, Y. S. Zhao, and C.-C. Kao, In. J. Hydrogen Energy 35, 11064 (2010).

${ }^{22}$ S. B. Wang, W. L. Mao, and T. Autrey, J. Chem. Phys. 131, 144508 (2009). 
${ }^{23}$ Y. Lin, W. L. Mao, and H.-K. Mao, Proc. Natl. Acad. Sci. USA 106, 8113 (2009).

${ }^{24}$ M. Ramzan and R. Ahuja, J. Phys. Chem. Solids 71, 1137 (2010).

${ }^{25}$ C. Murli and Y. Song, J. Phys. Chem. B 113, 13509 (2009).

${ }^{26}$ R. H. Crabtree, P. E. M. Siegbahn, O. Eisenstein, A. L. Rheingold, and T. F. Koetzle, Acc. Chem. Res. 29, 348 (1996).

${ }^{27}$ S. G. Shore and R. W. Parry, J. Am. Chem. Soc. 77, 6084 (1955).

${ }^{28}$ N. J. Hess, M. E. Bowden, V. M. Parvanov, C. Mundy, S. M. Kathmann, G. K. Schenter, and T. Autrey, J. Chem. Phys. 128, 034508 (2008).

${ }^{29}$ O. Gunaydin-Sen, R. Achey, N. S. Dalal, A. Stowe, and T. Autrey, J. Phys. Chem. B 111, 677 (2007).

${ }^{30}$ A. Paolone, O. Palumbo, P. Rispoli, R. Cantelli, and T. Autrey, J. Phys. Chem. C 113, 5872 (2009).

${ }^{31}$ N. J. Hess, G. K. Schenter, M. R. Hartman, L. L. Daemen, T. Proffen, S. M. Kathmann, C. J. Mundy, M. Hartl, D. J. Heldebrant, A. C. Stowe, and T. Autrey, J. Phys. Chem. A 113, 5723 (2009).

${ }^{32}$ H. Cho, W. J. Shaw, V. Parvanov, G. K. Schenter, A. Karkamkar, N. J. Hess, C. Mundy, S. Kathmann, J. Sears, A. S. Lipton, P. D. Ellis, and S. T. Autrey, J. Phys. Chem. A 112, 4277 (2008).

${ }^{33}$ S. M. Kathmann, V. Parvanov, G. K. Schenter, A. C. Stowe, L. L. Daemen, M. Hartl, J. Linehan, N. J. Hess, A. Karkamkar, and T. Autrey, J. Chem. Phys. 130, 024507 (2009).

${ }^{34}$ B. Håkansson, P. Andersson, and G. Bäckström, Rev. Sci. Instrum. 59, 2269 (1988).
${ }^{35}$ B. Sundqvist and O. Andersson, Phys. Rev. B 73, 092102 (2006).

${ }^{36}$ A. V. Talyzin, O. Andersson, B. Sundqvist, A. Kurnosov, and L. Dubrovinsky, J. Solid State Chem. 180, 510 (2007).

${ }^{37}$ B. Sundqvist, O. Andersson, and A. V. Talyzin, J. Phys. Condens. Matt. 19, 425201 (2007),

${ }^{38}$ V. Dmitriev, Y. Filinchuk, D. Chernyshov, A. V. Talyzin, A. Dzwilewski, O. Andersson, B. Sundqvist, and A. Kurnosov, Phys. Rev. B 77, 174112 (2008).

${ }^{39}$ O. Andersson, A. Soldatov, and B. Sundqvist, Phys. Rev. B 54, 3093 (1996).

${ }^{40}$ R. G. Ross, P. Andersson, B. Sundqvist, and G. Bäckström, Rep. Prog. Phys. 47, 1347 (1984).

${ }^{41}$ V. A. Konstantinov, V. G. Manzhelii, V. P. Revyakin, and S. A. Smirnov, Physica B 262, 421 (1999).

${ }^{42}$ R. C. Yu, N. Tea, M. B. Salamon, D. Lorents, and R. Malhotra, Phys. Rev. Lett. 68, 2050 (1992).

${ }^{43}$ H. Forsman and P. Andersson, J. Chem. Phys. 80, 2804 (1984).

${ }^{44}$ N. J. Hess, M. R. Hartman, C. M. Brown, E. Mamontov, A. Karkamkar, D. J. Heldebrant, L.L. Daemen, and T. Autrey, Chem. Phys. Lett. 459, 85 (2008).

${ }^{45}$ C. W. Garland and B. B. Weiner, Phys. Rev. B 3, 1634 (1971).

${ }^{46}$ R. G. Ross and O. Sandberg, J. Phys. C Solid State Phys. 12, 3649 (1979).

${ }^{47}$ H. Yurtseven and D. Kavruk, J. Mol. Struct. 924, 544 (2009). 